\title{
Acknowledgment to Reviewers of Biomechanics in 2021
}

\section{Biomechanics Editorial Office}

MDPI AG, St. Alban-Anlage 66, 4052 Basel, Switzerland

Rigorous peer-reviews are the basis of high-quality academic publishing. Thanks to the great efforts of our reviewers, Biomechanics was able to maintain its standards for the high quality of its published papers. The editors would like to extend their gratitude and recognition to the following reviewers for their precious time and dedication, regardless of whether the papers they reviewed were finally published:

Akiyama, Yasuhiro

Altoe, Alessandro

Andrysek, Jan

Angelillis, Marco

Barański, Robert

Barbosa, Alexandre

Barroso, Filipe

Benjaminse, Anne

Biz, Carlo

Borkowski, Paweł

Byeon, Haewon

Capelli, Claudio

Carnide, Filomena

Cecchi, Francesca

Cicchella, Antonio

Dizmen, Coskun Joe

Dumas, Raphaël

Favier, Clement

Felipe, José Luis

Fereidoonnezhad, Behrooz

Fleaca, Sorin Radu

García Aznar, Jose Manuel

Gomes, Beatriz

Gordon, Karen

Grouios, George

Gualdi-Russo, Emanuela

Herssens, Nolan

Hsu, Ching-Chi

Koh, Jae Chul

Kolesnikov, Gennady

Kong, Pui Wah

Konrad, Andreas

Kukla, Mateusz

Lee, Posen

Lee, Seo-Joon

Leirós-Rodríguez, Raquel
Lin, Che-Yu

Lisiński, Przemysław

Liu, Long

Loverro, Kari

Maranesi, Elvira

Marchena Rodríguez, Ana

Martín San Agustín, Rodrigo

Martínez-Jiménez, Eva María

McMahon, John J.

Mei, Qichang

Migliorini, Filippo

Moir, Gavin

Mok, Kam-Ming

Ngo, Chuong

Niu, Chuanxin

O'Sullivan, Rory

Pääsuke, Mati

Panday, Siddhartha

Peebles, Alexander

Peham, Christian

Pethick, James

Pop, Nicolae

Priego Quesada, Jose Ignacio

Räisänen, Anu

Roseiro, Luís

Sánchez-Sánchez, Javier

Santos, Paulo Cezar Rocha

Scano, Alessandro

Son, Jongsang

Tang, Lewei

Tang, Ruoliang

Vajda, Matej

Velepic, Marko

Ventura, Jessica

Villarejo Mayor, John Jairo 